\title{
Comparison of prenatal central nervous system abnormalities with postmortem findings in fetuses following termination of pregnancy and clinical utility of postmortem examination
}

https://doi.org/10.1515/jpm-2021-0501

Received October 8, 2021; accepted December 14, 2021;

published online December 31, 2021

\section{Abstract}

Objectives: In this study, we aimed to compare prenatal ultrasound (USG) and postmortem examination findings of central nervous system (CNS) abnormalities in fetuses following termination of pregnancy (TOP).

Methods: A total of 190 fetuses with USG-confirmed fetal CNS abnormalities of terminated pregnancies between January 2001 and January 2017 were retrospectively analyzed and USG and postmortem examination findings were compared.

Results: The most frequent CNS abnormalities were acrania/ anencephaly $(n=45,24 \%)$, spina bifida $(n=43,23 \%)$, and ventriculomegaly $(\mathrm{n}=35,18 \%)$. In 144 of the $190(76 \%)$ cases, there was total agreement between USG and postmortem examination diagnosis. Postmortem examination provided minor findings which did not change the major clinical diagnosis in two (1\%) cases with spina bifida and ventriculomegaly. In six (3\%) cases, the diagnosis changed after postmortem examination. In 25 of the 190 (13\%) cases with multiple abnormalities as evidenced by USG, CNS abnormality was unable to be confirmed at postmortem examination.

Conclusions: Our study results show an overall high agreement ( $76 \%$ ) between USG and postmortem examination findings for CNS malformations. Due to autolysis and fluid

*Corresponding author: Ozge Ozdemir, MD, Department of Obstetrics and Gynecology, Division of Maternal-Fetal Medicine, Istanbul University, Cerrahpasa Medical Faculty, Kocamustafapasa St. No: 53 34098, Istanbul, Turkey, Phone: +90 53263840 89,

E-mail: drozgeozde@gmail.com. https://orcid.org/0000-0003-28620802

Figen Aksoy, Department of Pathology, Istanbul University, Cerrahpasa Medical School, Istanbul, Turkey. https://orcid.org/ 0000-0003-4711-661X

Cihat Sen, Department of Obstetrics and Gynecology, Division of Maternal-Fetal Medicine, Istanbul University, Cerrahpasa Medical School, Istanbul, Turkey. https://orcid.org/0000-0002-2822-6840 structure, USG-confirmed CNS diagnosis cannot be always confirmed by postmortem examination. This potential discrepancy should be explained to patients before considering TOP. Postmortem examination is the gold standard to confirm prenatal diagnosis.

Keywords: central nervous system; congenital malformations; postmortem examination; prenatal ultrasound; termination of pregnancy.

\section{Introduction}

A prenatal ultrasound (USG) examination and a postmortem examination are the mainstays of a correct diagnosis in fetuses of terminated pregnancies due to congenital abnormalities $[1,2]$. The risk of false-positive diagnosis of congenital abnormalities is a major concern in prenatal diagnostics, particularly when termination of pregnancy (TOP) is an option. The definite diagnosis of major congenital abnormalities is of utmost importance to decide TOP. The confirmation of ultrasound findings is critical for the parents and the obstetrician, and for genetic counseling and epidemiological analysis [3]. The patient can decide to terminate pregnancy in case of false-positive diagnoses or, if the major malformation cannot be precisely detected, the patient may refuse TOP. Therefore, the accurate diagnosis is crucial for both patients and clinicians.

Major structural central nervous system (CNS) abnormalities can be easily diagnosed by prenatal USG examination [4]. About $30 \%$ of TOPs after the 12th week of gestation have been reported to be due to CNS abnormalities [5]. However, all abnormalities cannot be detected by USG examination, as many structures do not become sonographically apparent until the second and third trimester [6]. Confirmation of prenatal USG findings with postmortem examination is the gold standard to evaluate quality control and accuracy of the USG diagnosis $[1,7]$. The additional postmortem examination provides clinically relevant information and is a helpful instrument for complete assessment and optimal genetic counseling, 
leading to an accurate syndromic diagnosis or further genetic and/or chromosomal investigations [1].

In the literature, there is a limited number of studies investigating CNS abnormalities in perinatal postmortem examination populations [4, 8-11]. In this study, we aimed to compare prenatal ultrasound and postmortem examination findings of CNS abnormalities in fetuses following termination of pregnancy (TOP).

\section{Materials and methods}

This single tertiary care center, descriptive, retrospective study was conducted at the Department of Obstetrics and Gynecology, Division of Maternal-Fetal Medicine, Istanbul University-Cerrahpasa, Cerrahpasa Medical Faculty, between January 2001 and January 2017. Throughout the study period, there were 453 pregnancy terminations due to fetal anomaly. Among these cases, 190 (42\%) had CNS abnormalities. The USG and postmortem examination findings were compared. Due to the descriptive nature of the study, no statistical analysis was performed. A written informed consent was obtained from the parents before the TOP. The study protocol was approved by the institutional Ethics Committee (No. 227794). The study was conducted in accordance with the principles of the Declaration of Helsinki.

All prenatal USG examinations were performed using the Toshiba Xario Diagnostic USG System (Toshiba Medical Systems Corp., Otawara, Japan) between January 2001 and Aug 2016 and using the Voluson E10 USG machine (GE Healthcare Systems, WI, USA) after August 2016.

All patients with prenatal CNS malformation as evidenced by USG scan were referred to the Perinatology Council and assessed by the expert perinatologists. The option of TOP was offered to women with a gestational age of $<24$ weeks who had fatal CNS abnormalities in their fetuses or who had CNS abnormalities which would seriously affect the quality of life of the fetus.

All fetuses underwent postmortem examination after TOP. All postmortem examinations were carried out in the pathology department of our institution by a perinatal pathologist. All postmortem examinations including photography, whole body X-ray scan, gross examination, dissection, and histological examination of fetal organs were carried out in accordance with the Royal College of the Pathologists Postmortem Examination Guidelines [2]. The perinatal pathologist was unblinded to USG findings during the postmortem examination. The cases were divided into five groups as follows: (i) Group A, total agreement between USG and postmortem examination findings; (ii) Group B, anomaly detected with USG, but could not be confirmed at postmortem examination; (iii) Group C, postmortem examination confirmed all USG findings, but provided additional minor findings without changing the diagnosis; (iv) Group D, postmortem examination findings were consistent with prenatal USG, but additional major findings that changed the diagnosis were found; and (v) Group E, postmortem examination could not be performed due to autolysis.

\section{Results}

The median gestational week at the time of diagnosis was 18 (range, 13 to 24) weeks. The most frequent CNS abnormalities
Table 1: Number and types of central nervous system abnormalities of prenatal ultrasound $(n=190)$.

\begin{tabular}{lr}
\hline Ultrasonographic CNS abnormalities & Number of patients (\%) \\
\hline Acrania/anencephaly & $45(24)$ \\
Spina bifida & $43(23)$ \\
Ventriculomegaly & $35(18)$ \\
Encephalocele & $17(9)$ \\
Holoprosencephaly & $14(7)$ \\
Dandy-Walker malformation & $12(6)$ \\
Agenesis of corpus callosum & $7(4)$ \\
Cerebellar hypoplasia & $6(3)$ \\
Choroid plexus cysts & $3(2)$ \\
Arachnoid cyst & $1(0.5)$ \\
Lissencephaly & $1(0.5)$ \\
Iniencephaly + occipital encephalocele & $1(0.5)$ \\
Agenesis of cerebellar vermis & $1(0.5)$ \\
Schizencephaly & $1(0.5)$ \\
Cyst at posterior fossa & $1(0.5)$ \\
Mega cisterna magna & $1(0.5)$ \\
Diastematomyelia & $1(0.5)$ \\
Total & $190(100)$ \\
\hline
\end{tabular}

Data are given in number (\%), unless otherwise stated. CNS, central nervous system.

at USG scan were acrania/anencephaly $(45 / 190,23.7 \%)$, spina bifida (43/190, 22.6\%) and ventriculomegaly (35/190, 18.4\%). The distribution of USG findings is shown in Table 1.

The diagnosis at USG scan was compared with the postmortem examination findings in all cases. There was a total agreement between the USG and postmortem examination diagnosis in 144 of the $190(76 \%)$ cases (Group A, Figure 1). Twenty-five of the 190 (13\%) cases who had an abnormality at USG scan were not confirmed at the postmortem examination (Group B, Figure 1). The findings changed after postmortem examination in two of the 190 (1\%) cases in Group C and six of the 190 cases (3\%) in Group D. Postmortem examination confirmed all USG findings, but provided minor additional findings in 2/190 (1\%) cases in Group C. Both cases with 17 gestational week-spina bifida and 23 gestational week-ventriculomegaly were diagnosed with agenesis of corpus callosum additionally (Group C). Figure 1 shows the overall distribution of the cases into the five groups.

A detailed analysis of all cases in the study revealed the following findings: $100 \%$ of acrania cases was confirmed at postmortem examination. In 39 (90.6\%) of 43 spina bifida cases, postmortem examination confirmed the USG diagnosis. One $(2.3 \%)$ case was diagnosed with agenesis of corpus callosum additionally (Group C), while another $(2.3 \%)$ case with additional vertebra and thoracic defects was diagnosed with spondylothoracic dysostosis and the final two $(4.6 \%)$ cases at 16th and 17th gestational 


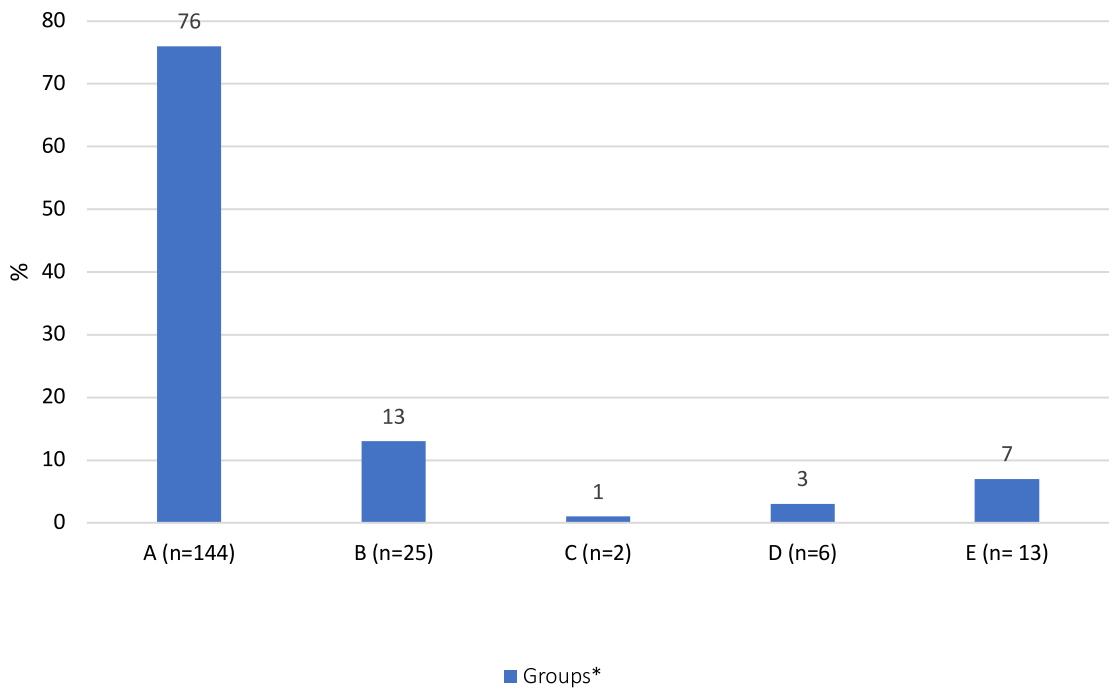

Figure 1: Classification according to agreement between ultrasound and postmortem examination. The total number of cases is 190. For the definition of groups, $A-E$, see methods section. week were diagnosed with amniotic band syndrome at postmortem examination based on additional findings (Table 2). Totally, 51.4\% (18/35) of ventriculomegaly cases were confirmed at postmortem examination, whereas USG findings could not be confirmed at postmortem examination in $16(45.7 \%)$ cases. One (2.8\%) case was diagnosed with posterior agenesis of corpus callosum additionally (Group C). Almost all cases with encephalocele were confirmed at postmortem examination (16/17). One encephalocele case at 15-gestational week (5.9\%) was diagnosed with Meckel-Gruber syndrome (MGS) with additional findings of polydactyly, polycystic kidney, proliferation at biliary duct at portal area, and ductal plate malformation (Table 2). Nearly all holoprosencephaly cases (13/14) were confirmed at postmortem examination. Only one $(7.1 \%)$ case was diagnosed with septo-optic dysplasia with additional major findings at postmortem examination (Table 2). Only 33.3\% (4/12) of the DandyWalker malformation (DWM) cases suspected at USG scan were confirmed at postmortem examination, and six $(50.0 \%)$ cases could not be confirmed due to autolysis, two (16.7\%) cases were assessed normal at postmortem examination. Among seven cases, three (42.9\%) agenesis of corpus callosum cases were confirmed at postmortem examination, one (14.2\%) case could not be confirmed at postmortem examination, three cases could not be confirmed due to autolysis. In six cases, cerebellar hypoplasia was diagnosed using USG, only one of them (16.7\%) was confirmed at postmortem examination, two (33.3\%) cases could not be confirmed at postmortem examination, and three (50.0\%) could not be assessed due to autolysis. Among three choroid plexus cases, only one (33.3\%) was confirmed at postmortem examination and one (33.3\%) case could not be assessed due to autolysis. Another
(33.3\%) case was unable to be confirmed at postmortem examination. Lissencephaly $(\mathrm{n}=1)$, schizencephaly $(\mathrm{n}=1)$, agenesis of cerebellar vermis $(\mathrm{n}=1)$, and iniencephaly + occipital encephalocele $(n=1)$ detected by USG were confirmed at postmortem examination (Figure 2).

\section{Discussion}

In the present study, we compared USG and fetal postmortem examination as diagnostic tools for the detection of structural fetal CNS abnormalities during the second trimester of pregnancy. Among 453 cases, CNS abnormalities were the most frequent in $42 \%(n=190)$ cases, consistent with previous studies [12-16]. Several studies comparing USG and postmortem examination findings have included all fetuses at any gestational week, spontaneous abortions and neonatal deaths; however, in the present study, only those who were in the second trimester of pregnancy were included.

In the present study, $76 \%(n=144)$ of all cases with ultrasonograhic diagnosis, CNS abnormality were totally confirmed by postmortem examination. This is consistent with the other studies $[3,11,17,18]$. In their study, Carroll et al. [11] found this ratio $77 \%$ in 61 fetuses with brain abnormality. In another study examining 144 fetal abnormalities during the seven-year period, the total agreement between the USG and postmortem examination diagnosis was $49 \%$ [17]. In a systematic review, Rossi et al. [8] found the agreement between the postmortem examination and prenatal USG to be $79.4 \%$ in fetuses with CNS abnormalities. In a long-term study, Struksnaes et al. [3] reported a correlation rate of full agreement as $90.1 \%$ for CNS abnormalities. 


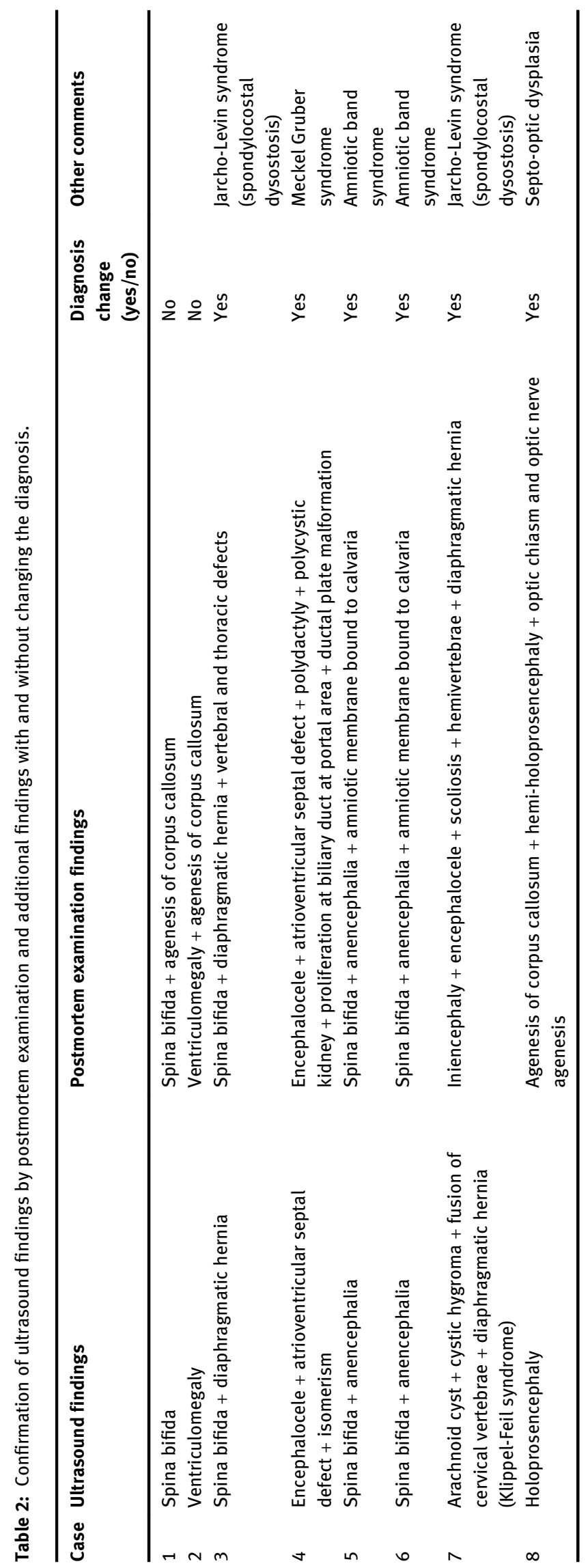




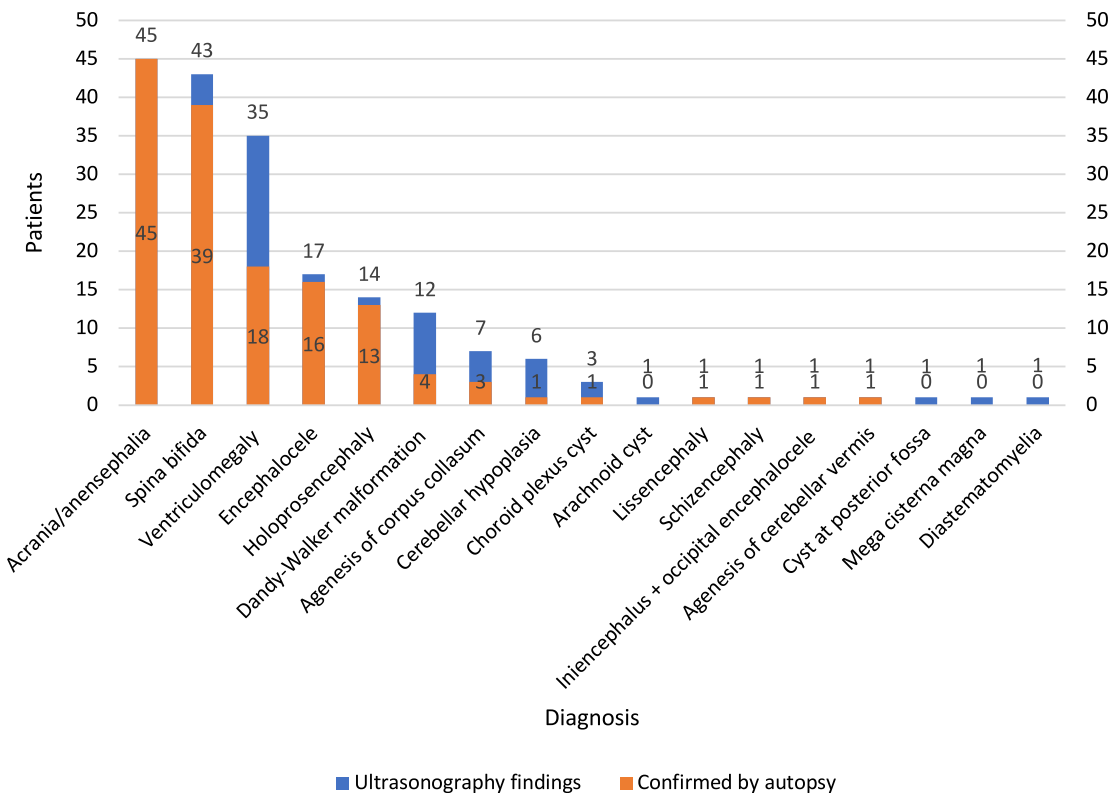

Figure 2: Ultrasound findings with total agreement and disagreement with postmortem examination.
In the present study, only 33\% (4/12) of DWM cases suspected at USG scan were confirmed at postmortem examination, and eight cases could not be confirmed, six of which were due to autolysis. This resulted in a relatively low confirmation rate. In the study of Struksnaes et al. [3], $55 \%$ (6/11) of the DWM cases were confirmed by postmortem examination. In the study of Carroll et al. [11], the most common disagreement was in DWM cases where only six (43\%) of 14 cases prenatally diagnosed with this condition had the same abnormality at postmortem examination. Concerning CNS, ventriculomegaly and small posterior fossa abnormalities such as DWM can collapse during postmortem examination and, thus, is difficult to confirm. In our study, a posterior fossa cyst in one case detected on prenatal USG was also found to be normal at postmortem examination and, therefore, we could not confirm the definite diagnosis.

There are many factors implicated in the correlation between USG and pathological diagnosis of DWM. First, the criteria used to define this complex are not strict and vary. Some of these criteria (e.g., dilatation of the fourth ventricle) may be easier to appreciate by USG or magnetic resonance imaging (MRI) than by anatomical dissection. In addition, anatomical relationships that may depend on the pressurized fluid in the fourth ventricle (e.g., rotation of the cerebellar vermis) may be lost during dissection. Thus, variants of DWM that are not associated with macroscopic or microscopic vermian dysgenesis can be overlooked at postmortem examination. In this study, we considered hypoplasia or aplasia of the vermis an obligate feature of DWM, in part, as there is a limited number of data suggesting that isolated dilatation of the fourth ventricle without associated vermian hypoplasia does not pose the same risk for functional neurological impairment as DWM complex.

Confirmation of the diagnosis at fetal postmortem examination may be challenging, as the ventricular spaces can be changed by autolysis or deformed artifactually. Previous studies have demonstrated disagreement between prenatal USG and postmortem examination for this anomaly. Additional radiological tests such as MRI to diagnose DWM or its variants prior to postmortem examination may be helpful. In a previous study, corpus callosum agenesis was evaluated by USG and MRI in 14 cases. Although a definitive diagnosis was made with prenatal USG in four cases, corpus callosum agenesis was diagnosed by MRI in all cases, 13 cases being complete and one case being partial agenesis [18].

Vimercati et al. [17] reported that autopsy could not confirm some brain malformations that were clearly detected at prenatal USG due to extensive postmortem autolysis, probably related to a long interval between fetal death and autopsy. The authors also emphasized that certain conditions of expulsion could hinder examination, as they involve an excessively long period of fetal retention leading to maceration in utero and tissue lysis, of brain tissue in particular.

Two spina bifida + anencephalia cases were diagnosed with amniotic band syndrome after postmortem examination. Spina bifida is sometimes confused with sacrococcygeal teratoma, isolated scoliosis/kyphosis or amniotic band syndrome [19]. Therefore, a prenatal diagnosis of 
spina bifida should be confirmed postnatally. Distinguishing spina bifida from the other abnormalities of the spine is important because these conditions have different causes and associated anomalies, and prognoses. With careful examination, the diagnosis of spina bifida is straightforward. The extent of the spinal dysraphism has been shown to extend above the visible lesion and the highest level per X-ray should be coded. It was reported the reasons of misdiagnosis of amniotic band syndrome were the sonographer not being familiar enough with this syndrome, a gastroschisis companying with bowl ectropion, a skull defect companying with encephalocele, spina bifida accompanying with spinal meningocele, fetal demise or decreasing fetus moving, the amniotic band only warping around fetal finger [19]. Rarely, a layer of the amniotic sac separates to form a ribbon-like strip called an amniotic band. Amniotic bands can prevent proper growth of any part of a developing fetus, a condition known as amniotic band disruption sequence. When the development of the brain, skull and/or scalp is interrupted by an amniotic band, the defect may resemble anencephaly; however, this condition is not a neural tube defect and rarely recurs in a subsequent pregnancy. Of note, anencephaly presence increases the risk of NTD in the future pregnancies. Therefore, this distinction is very important for counseling families [20]. Supplementation with $4 \mathrm{mg}$ folic acid per day resulted in a threefold reduction in NTD recurrence risk [20].

On the other hand, CNS abnormalities such as DWM and ventriculomegaly detected by prenatal USG cannot be always confirmed at postmortem examination. The potential benefits of postmortem examination, including details of the procedures and benefits in providing information on recurrence risks should be provided to parents. This is also helpful for counselling the affected couples, facilitating genetic studies to avoid such future events. Genetic counselling is of utmost importance, particularly in countries where the rates of consanguineous marriage and, thus, autosomal recessive diseases are high. In such cases, the recurrence of malformation is about $25 \%$ and, therefore, the diagnosis is critical to prevent recurrences. In our study, autosomal recessive diseases were diagnosed after postmortem examination findings in two cases. The first one was Jarcho-Levin syndrome, while the other was MGS. One case with encephalocele at the 19th gestational week was diagnosed with MGS. The sonographic characteristics depend on gestational age at MGS. It can be diagnosed before the 14th week of gestation. Later in the pregnancy, severe oligohydramnios makes it more difficult to establish the diagnosis by ultrasound alone. In these cases, postmortem examination is necessary to establish the diagnosis of MGS [21]. As there is $25 \%$ recurrence risk for
MGS, genetic counselling was provided to the affected couples. Furthermore, in one case terminated following USG-confirmed diagnosis of spina bifida, the diagnosis was changed to Jarcho-Levin syndrome based on the postmortem examination findings. Jarcho-Levin syndrome is an autosomal recessive disease, although some authors have described families with autosomal dominant inheritance [22]. Therefore, the family was provided genetic counselling before planning a new pregnancy.

Perinatal postmortem examination remains the gold standard to identify the cause of death and to elucidate all pathological findings [23, 24]. In recent years, a significant decline in the incidence of postmortem examination following TOP for prenatally detected fetal anomalies has been observed all over the world [25-27]. The decision of TOP due to fetal anomalies and giving consent for postmortem examination depends on ethnic, cultural, psychosocial, and religious factors. Some families do not give consent for TOP due to fetal malformation, while some others give consent for TOP, but refuse postmortem examination. So prenatal diagnosis cannot be confirmed by postmortem examination especially in the cases with abnormal fetal karyotype. Additionally, sonographic quality assurance cannot be confirmed. One of the reasons for the decline of postmortem examination is the unwillingness of the clinicians to offer it. In a study, $40 \%$ of clinicians did not offer the parents this opportunity for several reasons, i.e., the lack of availability of a perinatal pathologist, the belief that postmortem examinations do not useful new information on every case, a lack of formal training by clinicians in obtaining consent for a postmortem examination, and the thought that it is more difficult to discuss new information from the postmortem examination with bereaved parents [26]. Even in case of a postmortem examination not providing any additional information, the completion of the procedure confirming the clinical diagnosis offers comfort to both clinicians and parents [14].

The decreased use of postmortem examinations in developed countries has led to a rising demand for a lessinvasive alternative to the full perinatal postmortem examination. The MRI is the most promising candidate. There is a growing number of evidence in recent years suggesting that MRI is comparable to fetal postmortem examination in detecting fetal anomalies, particularly fetal brain abnormalities $[13,15]$. Recent studies validating postmortem MRI and comparing the findings with conventional perinatal postmortem examination have shown favorable agreement rates. However, there are still a significant number (up to $20 \%$ ) of patients who would benefit from the additional information provided by a conventional postmortem examination [24]. Although several studies have shown that 
postmortem MRI is an acceptable alternative to postmortem examination to most parents and healthcare providers, it remains clear that patients should be counseled that conventional postmortem examination is still the gold standard and can provide the most accurate information $[13,15]$.

On the other hand, the main disadvantage of postmortem imaging to formal postmortem examination is the loss of histological examination. As MRI imaging has high sensitivity and low specificity, biopsy is needed for the confirmation of the diagnosis [24]. In particular, MRI is useful for the evaluation of brain and spine findings at postmortem examination [15]. However, it is not costeffective and it may not available in every center. Although postmortem MRI has a definite role for fetal and neonatal cases, it is not clear whether it would be an adjunct to formal postmortem examination or whether it is the only investigation needed in selected cases [24]. However, MRI should be used before autopsy when ultrasound has diagnosed CNS abnormalities for which autopsy cannot confirm the presence, such as DWM, ventriculomegaly, cysts of the brain, and dilation of the third and fourth ventricles.

\section{Limitations}

Our perinatology center is a reference center where cases are referred throughout the country. Prenatal USG examinations are performed by experienced physicians and are discussed in the council with a multidisciplinary approach. In our study, the perinatal pathologist has a 15-year experience in postmortem examinations of fetuses of terminated pregnancies due to fetal anomalies. Nonetheless, there are some limitations to the present study. these results can not be generalized to national basis. This is the same for prenatal pathology practice.

\section{Conclusions}

In conclusion, this study showed an overall high degree of agreement between USG and postmortem examination findings of the fetuses following TOP in the second trimester of pregnancy. Although a high level of agreement between prenatal USG and postmortem examination findings was found for CNS abnormalities, postmortem examination still seems to be the most optimal tool to diagnose malformations and can often detect other abnormalities of clinical importance that are unable to be identified by USG. Therefore, it remains clear that conventional postmortem examination is the gold standard and can provide the most accurate information. Of note, clinicians should be aware of the limitations of postmortem examination in detecting fetal malformations in cases of retained macerated fetuses. In particular, DWM and ventriculomegaly cannot be confirmed at postmortem examination. Parents should be provided with full information about the potential disagreement between USG and postmortem examination. The role of the fetal postmortem examination is crucial to provide optimal genetic counselling and prevent recurrence.

Research funding: This study received no specific grant from any funding agency in the public, commercial or notfor-profit sectors.

Author contributions: 0.O., conceptualization, methodology, data collection, data analysis, writing; F.A. and C.S., supervision and review \& editing. All authors read and approved the final manuscript. All authors have accepted responsibility for the entire content of this manuscript and approved its submission.

Competing interests: The authors declare that they have no conflict of interest.

Informed consent: A written informed consent was obtained from each participant.

Ethical approval: This study was approved by the Istanbul University, Cerrahpasa Medical Faculty, Ethics Committee with the Approval No. 227794.

\section{References}

1. Antonsson P, Sundberg A, Kublickas M, Pilo C, Ghazi S, Westgren $M$, et al. Correlation between ultrasound and autopsy findings after 2nd trimester terminations of pregnancy. J Perinat Med 2008;36:59-69.

2. Royal College of Obstetricians and Gynaecologists and Royal College of Pathologists. Fetal and perinatal pathology. Report of a Joint Working Party. London: RCOG Press; 2001.

3. Struksnaes C, Blaas HG, Eik-Nes SH, Vogt C. Correlation between prenatal ultrasound and postmortem findings in 1,029 fetuses following termination of pregnancy. Ultrasound Obstet Gynecol 2016;48:232-8.

4. Struksnæs C, Blaas HK, Vogt C. Autopsy findings of central nervous system anomalies in intact fetuses following termination of pregnancy after prenatal ultrasound diagnosis. Pediatr Dev Pathol 2019;22:546-57.

5. Rouleau C, Gasner A, Bigi N, Couture A, Perez MJ, Blanchet P, et al. Prevalence and timing of pregnancy termination for brain malformations. Arch Dis Child Fetal Neonatal Ed 2011;96: F360-4.

6. Blaas HG, Eik-Nes SH. Sonoembryology and early prenatal diagnosis of neural anomalies. Prenat Diagn 2009;29:312-25.

7. Vogt C, Blaas HG, Salvesen KÅ, Eik-Nes SH. Comparison between prenatal ultrasound and postmortem findings in fetuses and 
infants with developmental anomalies. Ultrasound Obstet Gynecol 2012;39:666-72.

8. Rossi AC, Prefumo F. Correlation between fetal autopsy and prenatal diagnosis by ultrasound: a systematic review. Eur J Obstet Gynecol Reprod Biol 2017;210:201-6.

9. Isaksen CV, Eik-Nes SH, Blaas HG, Torp SH. Comparison of prenatal ultrasound and postmortem findings in fetuses and infants with central nervous system anomalies. Ultrasound Obstet Gynecol 1998;11:246-53.

10. Melcer Y, Maymon R, Krajden Haratz K, Goldrat I, Shavit M, BenAmi I, et al. Termination of pregnancy due to fetal central nervous system abnormalities performed after 24 weeks' gestation: survey of 57 fetuses from a single medical center. Arch Gynecol Obstet 2018;298:551-9.

11. Carroll SG, Porter H, Abdel-Fattah S, Kyle PM, Soothill PW. Correlation of prenatal ultrasound diagnosis and pathologic findings in fetal brain abnormalities. Ultrasound Obstet Gynecol 2000;16:149-53.

12. Grover S, Garg B, Sood N, Arora K. Lethal congenital malformations in fetuses-antenatal ultrasound or perinatal autopsy. Fetal Pediatr Pathol 2017;36:220-31.

13. Shelmerdine SC, Hutchinson JC, Arthurs OJ, Sebire NJ. Latest developments in post-mortem foetal imaging. Prenat Diagn 2020; 40:28-37.

14. Neşe N, Bülbül Y. Diagnostic value of perinatal autopsies: analysis of 486 cases. J Perinat Med 2018;46:175-81.

15. Griffiths PD, Paley MN, Whitby EH. Post-mortem MRI as an adjunct to fetal or neonatal autopsy. Lancet 2005;365:1271-3.

16. Boyd PA, Tondi F, Hicks NR, Chamberlain PF. Autopsy after termination of pregnancy for fetal anomaly: retrospective cohort study. BMJ 2004;328:137.

17. Vimercati A, Grasso S, Abruzzese M, Chincoli A, de Gennaro A, Miccolis $\mathrm{A}$, et al. Correlation between ultrasound diagnosis and autopsy findings of fetal malformations. J Prenat Med 2012;6: 13-7.

18. d'Ercole C, Girard N, Cravello L, Boubli L, Potier A, Raybaud C, et al. Prenatal diagnosis of fetal corpus callosum agenesis by ultrasonography and magnetic resonance imaging. Prenat Diagn 1998;18:247-53.

19. Niu J. Amniotic band syndrome: the reason for missed diagnosis. Poster presentation at 28th World Congress on Ultrasound in Obstetrics and Gynecology, EP11.17. Ultrasound Obstet Gynecol 2018;52:248.

20. Avagliano L, Massa V, George TM, Qureshy S, Bulfamante GP, Finnell RH. Overview on neural tube defects: from development to physical characteristics. Birth Defects Res 2019;111:1455-67.

21. Alexiev BA, Lin X, Sun CC, Brenner DS. Meckel-Gruber syndrome: pathologic manifestations, minimal diagnostic criteria, and differential diagnosis. Arch Pathol Lab Med 2006;130:1236-8.

22. Jarcho S, Levin P. Hereditary malformation of the vertebral bodies. Bull Johns Hopkins Hosp 1938;62:216-26.

23. Phillips JJ, Mahony BS, Siebert JR, Lalani T, Fligner CL, Kapur RP. Dandy-Walker malformation complex: correlation between ultrasonographic diagnosis and postmortem neuropathology. Obstet Gynecol 2006;107:685-93.

24. Ernst $L M$. A pathologist's perspective on the perinatal autopsy. Semin Perinatol 2015;39:55-63.

25. Oliver E, Rood KM, Ma'ayeh M, Berghella V, Silver RR. Still birth and fetal postmortem examination rates in the United States: analysis of fetal death certificates [350]. Obstet Gynecol 2020;135:166S.

26. Rose C, Evans M, Toopey J. Falling rates of perinatal postmortem examination: are we to blame? Arch Dis Child Fetal Neonatal Ed 2006;91:F465.

27. Gordijn SJ, Erwich JJ, Khong TY. The perinatal autopsy: pertinent issues in multicultural Western Europe. Eur J Obstet Gynecol Reprod Biol 2007;132:3-7. 